\title{
EXTENSIONS OF MAPS AS FIBRATIONS AND COFIBRATIONS
}

\author{
BY \\ FRANK QUINN( $\left.{ }^{1}\right)$
}

\begin{abstract}
Suppose $f: X \rightarrow Y$ is a map of 1 -connected spaces. In the "stable" range, roughly where the connectivity of $Y$ exceeds the homology, or homotopy, dimension of $X$, it is well known that $f$ can be extended as a cofibration $C \rightarrow X \rightarrow Y$, or respectively a fibration $X \rightarrow Y \rightarrow B$. $A$ criterion is given for the existence of such extensions in a less restrictive "metastable" range. A main result is that if $f$ is at least 2-connected and 2 con $Y \geq \operatorname{dim} Y-1, \operatorname{dim} X$, then $f$ extends as a cofibration if and only if the map $(1 \times f) \Delta: X \rightarrow(X \times Y) / X$ factors through $f_{\bullet}$
\end{abstract}

We consider the question: Given a map $f: X \rightarrow Y$, when can it be extended up to homotopy to a fibration $X \rightarrow Y \rightarrow B$, or a cofibration $C \rightarrow X$ $\rightarrow Y$ ? Generally no such extension is possible. In an appropriate "stable" range of dimensions and connectivities the extension can be made. The object of this paper is to give a necessary and sufficient condition for the extension in the "metastable" range.

Only the simply connected version is considered here, and spaces are understood to have the homotopy type of a CW complex; basepoints are nondegenerate. A cofibration lemma similar to 1.2 was announced in [3], and the nonsimply connected version is given in [4]. It was developed for use as a main step in constructing a surgery theory for Poincaré spaces. Corollary 1.3 and some standard Spanier-Whitehead duality can be used to do surgery on simply connected Poincaré spaces.

T. Ganea [2] and R. Nowlan [6] have similar results, but their extension criterion involves operation, or "cooperation" of an h-space, or co-h-space. Our criterion is based, in the cofibration case, on a homotopy analog of the vanishing of certain products in cohomology. See the comments after 1.3.

1. Statements of results. First some notations are established. For a map $f: X \rightarrow Y$ we denote by $Y$, the range of $f$ converted functorially into

Received by the editors February 18, 1974 and, in revised form, August 26, 1974. AMS (MOS) subject classifications (1970). Primary 55D05.

Key words and phrases. Fibration, cofibration, metastable extension.

(1) Partially supported by NSF-GP 43831. 
a cofibration (mapping cylinder of $f$ ), and $X^{f}$ the domain of $f$ made into a fibration (path space construction). The fiber and cofiber of $f$ are then given respectively by $\left.X^{f}\right|_{*}$ and $Y_{f} \wedge X$.

We wish to make perfectly clear what we mean by connectivity and dimension. Suppose $f: X \rightarrow Y$ is a map of connected spaces. Define

$$
\begin{aligned}
& \operatorname{con} f=\max \left\{k \mid \pi_{j}\left(Y_{f}, X\right)=0, j \leq k\right\}, \\
& \operatorname{dim}_{\pi} f=\min \left\{k \mid \pi_{j}\left(Y_{f}, X\right)=0, j>k\right\}, \\
& \operatorname{dim}_{H} f=\min \left\{k \mid H_{j}\left(Y_{f}, X\right)=0, j>k\right\}, \\
& \operatorname{dim} f=\min \left\{k \mid H_{j}\left(Y_{f}, X\right)=0, \text { and } H^{j}\left(Y_{f}, X ; A\right)=0, \text { any } A, j>k\right\} .
\end{aligned}
$$

The main result is

1.1. Cofibration. If $f: X \rightarrow Y$ is at least 2-connected, $X, Y$ 1-connected, and $\operatorname{dim} f \leq 2$ con $Y+\operatorname{con} f$, then $f$ extends up to bomotopy to a cofibration if and only if the map $X \rightarrow X /\left[\left.X^{f}\right|_{*}\right]$ factors through $f$.

Fibration. If $f: X \rightarrow Y$ is a map with $\operatorname{dim}_{\pi} f<2$ con $X+\operatorname{con} f$, then $f$ extends as a fibration if and only if the map $\left.Y^{q}\right|_{*} \rightarrow Y$ factors through $f$, where $q: Y \rightarrow Y_{f} / X$ is the quotient map.

This will be proved in $\$ 3$. There is a uniqueness which asserts that with the dimension restriction lowered by 1 , the cofibration or fibration is uniquely determined by the factoring. In the "stable" range the factoring is automatic.

In the cofibration case the factoring hypothesis can be made more explicit.

1.2. Corollary. If $f: X \rightarrow Y$ is a map, at least 2-connected, of 1-connected spaces, and 2 con $Y \geq \operatorname{dim} Y-1, \operatorname{dim} X$ then $f$ extends as a cofibration if and only if the map $(1 \times f) \Delta: X \rightarrow(X \times Y) / X$ factors through $f$.

Proof. Since $\operatorname{dim} f \leq \operatorname{dim} Y, \operatorname{dim} X+1$, and con $f \geq \operatorname{con} Y, \operatorname{con} X+1$, the inequality of 1.1 is implied by $\operatorname{dim} Y, \operatorname{dim} X+1 \leq 3 \operatorname{con} Y, 2$ con $Y+$ con $X+1$. The inequality of 1.2 certainly implies this.

The dimension restriction implies that $Y$ is a suspension. In this case $X /\left[\left.X^{f}\right|_{*}\right]$ is homotopy equivalent to $\left[\left.X^{f}\right|_{*}\right]_{+} \wedge Y[5$, p. 455], and the composition

$$
X \rightarrow X /\left[\left.X^{f}\right|_{*}\right] \simeq\left[\left.X^{f}\right|_{*}\right]_{+} \wedge Y \rightarrow X_{+} \wedge Y
$$

is homotopic to $(1 \times f) \Delta$. Therefore to recover the hypotheses of 1.1 from 1.2 , we need the connectivity of the map $\left[\left.X^{f}\right|_{x}\right]_{+} \wedge Y \rightarrow X_{+} \wedge Y$ to be greater than or equal to $\operatorname{dim} Y$ and $\operatorname{dim} X+1$. This connectivity is 2 con $Y+1$, which gives the inequality of 1.2.

The important special case $Y=S^{m}$ is covered in more detail.

1.3. Corollary. Suppose $f: X \rightarrow S^{m}$ is at least 2-connected, and $m \geq$

2. If $m=2$ and $\operatorname{dim} X \leq 4$, or $\operatorname{dim} X \leq 3 m-4,2 m+\operatorname{con} X-2$, and a null. 
bomotopy of $(f \wedge 1) \Delta: X \rightarrow S^{m} X$ is given, then there is a secondary obstruction map $X \rightarrow s^{2 m-1}\left(X_{+}\right)$defined. If this map is nullhomotopic, $f$ extends as a homotopy cofibration.

The conclusion of 1.3 should be interpreted as "removing a cell" from $X$. Given a cofibration $C \rightarrow X \rightarrow S^{m}$, the Hurewicz theorem applied to $(X, C)$ using $H_{*}(X, C) \approx H_{*}\left(S^{m}\right)$ provides a map $P: S^{m-1} \rightarrow C$ and a homotopy equivalence $C U_{P} D^{m} \simeq X$. On the other hand if $a \in H^{m}(X)$ is the cohomology class $f^{*}\left[S^{m}\right]$, a first obstruction to killing this cohomology class by removing a cell is that all products $a \cap k$ should vanish for $k \in H_{j}(X)$, $j>0$. This is the algebraic analog of $(f \wedge 1) \Delta$, since this map induces the homomorphism an on homology. Further, given that all products with $a$ vanish, secondary cohomology operations can be defined. The essential one is induced by the obstruction map $X \rightarrow S^{2 m-1} X$ in the statement of 1.3. Naturally the geometric conditions are much stronger than the cohomology analogs.

Proof of 1.3. The inequalities are those of 1.1. $Y=S^{m}$ so $\left.X /\left.X^{f}\right|_{*}\right] \simeq$ $S^{m}\left[\left.X^{f}\right|_{*}\right] \vee S^{m}$. The map of $X$ into this space factors through $f$ if and only if the first factor is nullhomotopic, because $\left[\left.X^{f}\right|_{*}\right]$ is at least 1 -connected. As in 1.2, the composition $X \rightarrow S^{m}\left[\left.X^{f}\right|_{*}\right] \rightarrow S^{m} X$ is $(f \wedge 1) \Delta$, and we have assumed a nullhomotopy of this is given. The nullhomotopy defines a lift of $X$ to the fiber of $S^{m}\left[\left.X^{f}\right|_{*}\right] \rightarrow S^{m} X$, which we can identify. There is a cofibration

$$
\left.X^{f}\right|_{*} \rightarrow X^{f} \rightarrow X^{f} /\left[\left.X^{f}\right|_{*}\right] \simeq S^{m} \wedge\left[\left.X^{f}\right|_{*}\right]_{+}
$$

This suspends to a cofibration

$$
S^{2 m-1}\left(\left[\left.X f\right|_{*}\right]_{+}\right) \rightarrow S^{m}\left[\left.X^{f}\right|_{*}\right] \rightarrow S^{m} X .
$$

According to 2.2 , this sequence is also a fibration up to dimension $3 m+$ con $X-1$. Further the map $\left.s^{2 m-1}\left(\left.X^{f}\right|_{*}\right]\right) \rightarrow s^{2 m-1}\left(X_{+}\right)$is $3 m-2$ connected. Since both of these numbers exceed $\operatorname{dim} X$, the lift defines a map $X \rightarrow$ $s^{2 m-1}\left(X_{+}\right)$. This map is nullhomotopic if and only if there is a nullhomotopy of $X \rightarrow S^{m}\left[\left.X^{f}\right|_{*}\right]$ covering the given homotopy of $(f \wedge 1) \Delta .1 .3$ now follows from 1.1 .

The fibration case lacks the corresponding calculation of $\left.Y^{q}\right|_{*^{*}}$ If the extension as a fibration is possible, it can be calculated via [1, Theorem 1.1], but there does not seein to be an a priori understanding at present. Significant applications are also lacking, in part because of the scarcity of finite Postnikov systems in nature.

2. The stable case. We first make more precise what is meant by a fibration or cofibration "up to homotopy". 
2.1. Definition. Suppose there is a sequence of maps $X \stackrel{f}{\rightarrow} Y \stackrel{g}{\rightarrow} Z$ together with a nullhomotopy of the composition $g \circ f$.

(i) They form a homotopy fibration if the induced map $\left.X \rightarrow Y^{8}\right|_{*}$ is a homotopy equivalence. They form an $n$-fibration if $\left.X \rightarrow Y^{8}\right|_{*}$ is n-connected.

(ii) Similarly they form a homotopy cofibration (resp. $n$-cofibration) if $Y_{f} / X \rightarrow Z$ is a homotopy equivalence (resp. $n$-connected).

The first step is to specify to what extent these situations imply each other.

2.2. Proposition. Consider a sequence of 1-connected spaces $X \stackrel{\perp}{\rightarrow}$ $\stackrel{8}{\rightarrow}$ together with a nullhomotopy of $\mathrm{g} \circ \mathrm{f}$.

(i) If they form a bomotopy fibration, then they are also a con $f+c o n$ cofibration.

(ii) If a homotopy cofibration, then also a con $f+\operatorname{con} X$-fibration.

Proof. Part (i) results from applying the corollary of the homotopy excision theorem [5, p. 487] to $Y, X \rightarrow Y, / X, *$, and using the isomorhpism $\pi_{*}(Y, X) \simeq \pi_{*}(Z, *)$ of a homotopy fibration. Similarly for (ii), to find the connectivity of $\left.X \rightarrow Y^{8}\right|_{*}$, go to $\left(Y_{f}, X\right) \rightarrow\left(Y^{8},\left.Y^{8}\right|_{*}\right)$ and use the isomorphism $\pi_{*}\left(Y^{g},\left.Y^{8}\right|_{*}\right) \simeq \pi_{*}(Z, *) \simeq \pi_{*}(Y, / X)$. Again the excision theorem applies to $\left(Y_{f}, X\right) \rightarrow\left(Y_{f} / X, *\right)$.

Next is the stable version of 1.1 .

2.3. Lemma. Let $f: X \rightarrow Y$ be a map of 1 -connected spaces. If con $f$ $\geq 2$ and $\operatorname{dim}_{H} f \leq \operatorname{con} f+\operatorname{con} Y$, or $\operatorname{dim}_{\pi} f \leq \operatorname{con} f+\operatorname{con} X$, then $f$ can be extended as a bomotopy cofibration or fibration respectively. If the inequality is strict, then this extension is unique up to bomotopy.

Proof. Consider the homotopy fibration $\left.X^{f}\right|_{*} \stackrel{i}{\rightarrow} X \stackrel{f}{\rightarrow} Y .\left.X^{f}\right|_{*}$ is 1 connected since $f$ is 2 -connected. By 2.2 , this is a con $i+\left.\operatorname{con} X^{f}\right|_{*}+1=$ con $Y+\operatorname{con} f$-cofibration. Truncation of the Moore tower of $\left.X^{f}\right|_{*}$ at this level gives a map $\left.C \rightarrow X^{f}\right|_{*}$ which is an isomorphism on $H_{j}, j \leq \operatorname{con} Y+$ con $f$, and with $H_{j}(C)=0$ for $j>$ con $Y+\operatorname{con} f$. Now if $\operatorname{dim}_{H} f \leq \operatorname{con} Y+$ con $f$, then $X / C \rightarrow Y$ induces an isomorphism on homology. Thus $C \rightarrow X \rightarrow$ $Y$, with the nullhomctopy from the composition $\left.C \rightarrow X^{f}\right|_{*} \rightarrow X \rightarrow Y$, is a homotopy cofibration.

For the uniqueness statement, consider another extension $D \rightarrow X \rightarrow Y$. The nullhomotopy defines a lift $\left.D \rightarrow X^{f}\right|_{*}$, and provides a homology con $Y$ $+\operatorname{con} f$-truncation of $\left.X^{f}\right|_{*}$. If $\operatorname{dim}_{H} f+1 \leq \operatorname{con} Y+\operatorname{con} f_{g}=j$, then $H_{j}(D)=$ $H_{j}(C)=0$, and the truncaticn is unique. Thus $C \simeq D$.

The proof of the fibration statement is similar, truncating the Postnikov tower of $Y, / X$ at dimension $\operatorname{con} f+\operatorname{con} X$. 
3. Proof of the main result 1.1. The cofibration theorem is proved in detail. It involves an induction: the problem for $f: X \rightarrow Y$ is reduced to the problem for $k: F \rightarrow Z$ where $\operatorname{dim} f=\operatorname{dim} k, \operatorname{con} f=\operatorname{con} k$, but $\operatorname{con} Z>\operatorname{con} Y$. By repeating this construction the inequality $\operatorname{dim}_{H} k \leq \operatorname{con} Z+\operatorname{con} f$ is eventually achieved, and 2.3 applies to give a solution.

Let $F$ denote the truncation of the Moore tower of $\left.X^{f}\right|_{*}$ in dimension $\operatorname{dim} f-1$. The hypothesized factoring of $X \rightarrow X /\left[\left.X^{f}\right|_{*}\right]$ gives a commutative diagram:

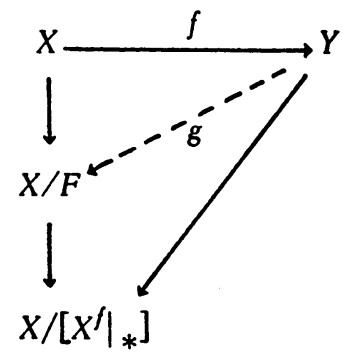

Obstructions to constructing the dotted lift lie in

$$
H^{i}\left(Y_{f}, X ; \pi_{i}\left(X /\left[\left.X^{f}\right|_{*}\right], X / F\right)\right) \text {. }
$$

The coefficient group is 0 for $i \leq \operatorname{dim} f$ by definition of $F$, and the cohomology group vanishes above $\operatorname{dim} f$ by definition of $\operatorname{dim} f$. Thus the lift $g$ exists making the diagram commute.

Now $g: Y \rightarrow X / F$ has $\operatorname{dim}_{H} g=\operatorname{dim}_{H} f$, con $g=\operatorname{con} Y+\operatorname{con} f$, by 2.2, and $\operatorname{con} X / F=\operatorname{con} Y$. We have assumed

$$
\operatorname{dim}_{H} g=\operatorname{dim}_{H} f \leq \operatorname{con} f+2 \operatorname{con} Y=\operatorname{con} g+\operatorname{con}(X / F),
$$

so Lemma 2.3 extends $g$ backwards as a cofibration.

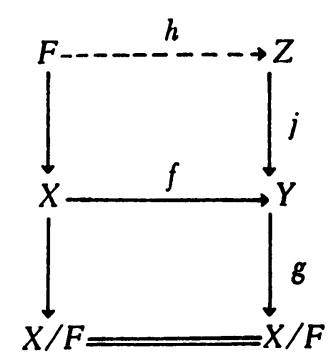

$(j, g)$ is a homotopy $\operatorname{dim}_{H} f$-fibration, so the lift $b$ of $f$ exists making the diagram homotopy commute.

Now consider $h$. By extending the rows to the right as cofibrations we see that $Z_{b} / F \approx Y_{f} / X$. Thus $\operatorname{con} f=\operatorname{con} b, \operatorname{dim} f=\operatorname{dim} b$ since these are 
invariants of the cofiber. If $b$ can be extended as a cofibration, this extension gives an extension for $f$ also. Finally

$$
\operatorname{con} Z=\operatorname{con} b-1=\operatorname{con} f+\operatorname{con} Y-1 \text {. }
$$

Since $\operatorname{con} f \geq 2$, this will complete the induction step.

The factoring hypothesis must be verified for $h$. Form the diagram

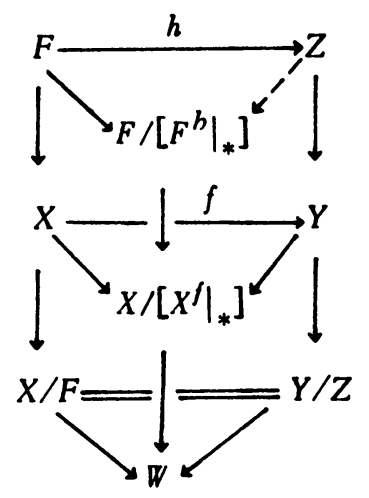

where the front column is a cofibration.

The front column is a con $\left.F /\left[\left.F^{b}\right|_{*}\right]+\operatorname{con}\left(F /\left[\left.F^{h}\right|_{*}\right] \rightarrow X /\left.X^{f}\right|_{*}\right]\right)=$ 2 con $Y+\operatorname{con} f$-fibration, again by 2.2 . Since $\operatorname{dim} b \leq 2 \operatorname{con} Y+\operatorname{con} f$, the indicated dotted lift exists.

The proof of the fibration statement is essentially dual to the above.

\section{BIBLIOGRAPHY}

1. T. Ganea, A generalization of the homology and homotopy suspension, Comment. Math. Helv. 39 (1964), 295-322. MR 31 \#4033.

2. - Induced fibrations and cofibrations, Trans. Amer. Math. Soc. 127 (1967), 442-459. MR $35 \# 1025$.

3. F. Quinn, Surgery on Poincaré and normal spaces, Bull. Amer. Math. Soc. 78 (1972), 262-267. MR $45 \# 6014$.

4. - Poincaré spaces (in preparation).

5. E. H. Spanier, Algebraic topology, McGraw-Hill, New York, 1966. MR 35 $\# 1007$.

6. R. A. Nowlan, $A_{n}$-actions on fibre spaces, Indiana Univ. Math. J. 21 (1971/72), 285-313. MR 44 \#5963.

DEPARTMENT OF MATHEMATICS, YALE UNIVERSITY, NEW HAVEN, CONNECTICUT 06520 